\title{
On time's arrow in Ehrenfest models with reversible deterministic dynamics
}

\author{
R. Metzler and W. Kinzel \\ Institut für Theoretische Physik, Universtität Würzburg, Am Hubland, D-97074 Würzburg, Germany \\ I. Kanter \\ Minerva Center and Department of Physics, Bar Ilan University, 52900 Ramat Gan, Israel
}

\begin{abstract}
We introduce a deterministic, time-reversible version of the Ehrenfest urn model. The distribution of first-passage times from equilibrium to non-equilibrium states and vice versa is calculated. We find that average times for transition to non-equilibrium always scale exponentially with the system size, whereas the time scale for relaxation to equilibrium depends on microscopic dynamics. To illustrate this, we also look at deterministic and stochastic versions of the Ehrenfest model with a distribution of microscopic relaxation times.
\end{abstract}

\section{INTRODUCTION}

Physical laws of motion are time reversible. As a consequence, from a movie of a few interacting particles one cannot distinguish whether the movie is running forward or backward. This seems to be different when we observe a very large number of particles. Macroscopic properties like the density of particles show a direction of time. If the milk in the coffee shrinks to a single drop we know that the movie is running backward.

More than a hundred years ago Ludwig Boltzmann gave convincing arguments for the irreversible macroscopic behaviour of particles moving with reversible microscopic laws. The fraction of initial states which leads to reversible macroscopic behaviour is so extremely small that we will never be able to observe it. With probability one, we observe a time's arrow in macroscopic properties.

Hence, through the selection of the initial state, the deterministic motion receives probabilistic elements. In fact describing a large system of interacting particles by either a stochastic motion (Boltzmann transport equations) or ensemble theory (statistical mechanics) turned out to be very successful.

However, the success of Boltzmann's ideas did not suppress the discussion about the foundations of irreversible properties, even hundred years later [1 5 . For example, there was a round table discussion on this subject on the STATPHYS 98 conference [6,7], where Prigogine introduced novel microscopic laws which are irreversible with time. One reason for this ongoing discussion is the absence of rigorous mathematical proofs of irreversible properties in the thermodynamical limit. Furthermore, solvable models where irreversible macroscopic properties can be well defined and investigated almost do not exist, to our knowledge. Either one studies stochastic models like the famous one of Paul and Tanja Ehrenfest [8], or disorder averages as for the Kac ring [9], or ensembles of chaotic noninteracting particles as in the Lorentz gas [10,11]. However, a single trajectory of interacting particles is difficult to investigate in this context.

Numerical simulations of the equations of motions do not help much in this context, either. The algorithms can be formulated in a time reversible way and the de- cay of Boltzmann's H-function has been demonstrated [12]. However, the state space is too large to study any details, for instance to estimate the fraction of untypical inital states or to find the time scale of the Poincaré return time.

One should keep in mind that we want to understand the property of a single trajectory for a short time. Hence, ensemble averages do not give a basic explanation of irreversible properties, since they contain an average over infinitely many trajectories. Ergodic theory does not help either, since it needs time averages over infinitely large times (at least up to the Poincaré return time) whereas real physical systems or computer simulations run over time scales which are extemely short compared to the return time.

In this paper we introduce a model with deterministic time reversible dynamics which can be analysed in detail. It is a deterministic extension of the Ehrenfest model discussed in Section IV. The dynamics is ergodic, each state is visited once during a cycle. The Poincaré return time is known exactly and the Boltzmann and Gibbs entropies can be defined precisely. From numerical calculations we obtain the distribution of first passage times the system needs to go from a non equilibrium state to a state with largest Boltzmann entropy as well as in the reverse direction. The scaling of average first passage times with system size is calculated. Finally we compare the results of the deterministic model with the corresponding ones of the stochastic version of the Ehrenfest model.

\section{DETERMINISTIC EHRENFEST MODEL: SHIFT REGISTER GENERATOR}

The microscopic state $x$ of our model has $N$ binary variables $x_{i} \in\{0,1\} ; i=1, \ldots, N$,

$$
x(t)=\left(x_{1}, x_{2}, \ldots, x_{N}\right) .
$$

The time $t$ is discrete. At each time step a new bit $x_{0}$ is generated and replaces $x_{1}$, whereas the old bits $x_{1}, \ldots, x_{N-1}$ are moved one step to the right: 


$$
\begin{gathered}
x_{1} \rightarrow x_{2} \\
\vdots \\
x_{N-1} \rightarrow x_{N} \\
x_{0} \rightarrow x_{1} .
\end{gathered}
$$

The last bit $x_{N}$ is deleted. The new bit $x_{0}$ is constructed using the theory of primitive polynomials modulo two which is also used to generate pseudo random numbers 13 16. If $x_{0}$ is the sum modulo two of a few bits $x_{i}$ at certain positions $i$, then any initial state $x(0)$ runs through all possible states $x$ except the state zero $(0,0, \ldots, 0)$. For instance for $N=97$ one finds from the table of Ref. |14:

$$
x_{0}=\left(x_{97}+x_{6}\right) \bmod 2 .
$$

In our investigation we use such sequences with maximal cycle length, taking the generators from 14 for $N<100$ and from 16] for higher $N$.

For the macroscopic property $M(x)$, which we will investigate, we take the number of $x_{i}=1$,

$$
M(x)=\sum_{i=0}^{N} x_{i}
$$

Note that at each time step $M$ changes by \pm 1 at most. The original Ehrenfest model considered $N$ balls in two urns. $x_{i}=0(1)$ means that ball $i$ is in the left (right) urn. $M$ is the number of balls in the right urn. In the Ehrenfest model the balls were chosen randomly. Here we define a deterministic rule to move the particles between the two urns.

The equations of motion (2) and (3) are time reversible; for instance from $(3)$ one finds

$$
x_{97}=\left(x_{0}+x_{6}\right) \bmod 2 \text {. }
$$

Hence, from the first and seventh bit of state $x(t+1)$ one calculates the $97^{t h}$ bit of $x(t)$, and a shift to the left gives the rest of $x(t)$.

We have now defined a model where time and states are discrete. It has the following properties:

1. The equations of motion are deterministic and time reversible.

2. Each initial state returns to itself after $2^{N}-1$ time steps; i.e. the Poincaré return time is $T_{R}=2^{N}-1$.

3. The Boltzmann entropy $S_{B}$ is given by the number of microstates $x$ which have the macroscopic property $M$. Since the distribution of macrostates is the binomial distribution (with the exception of the zero state),

$$
p(M(x))=\left(\begin{array}{c}
N \\
M(x)
\end{array}\right) /\left(2^{N}-1\right),
$$

one finds for the entropy in dimensionless units:

$$
S_{B}(x)=\ln \left(\begin{array}{c}
N \\
M(x)
\end{array}\right) .
$$

4. Since each state $x$ is visited once during each cycle, it has an identical statistical weight in a Gibbs ensemble. Hence, the Gibbs entropy $S_{G}$ is given by

$$
S_{G}=\ln \left(2^{N}-1\right) \simeq N \ln 2 .
$$

5. The system is ergodic. When the system is observed over the return time $T_{R}$, the time average $\bar{M}$ agrees with ensemble average $\langle M\rangle$ :

$$
\bar{M}=\frac{1}{T_{R}} \sum_{t>0}^{T_{R}} M[x(t)]=\langle M\rangle=\frac{N}{2} .
$$

6. The most probable value of $M$ agrees with time and ensemble average,

$$
M_{m p}=\bar{M}=\langle M\rangle=\frac{N}{2} .
$$

There are

$$
\left(\begin{array}{c}
N \\
N / 2
\end{array}\right) \simeq \sqrt{\frac{2}{\pi}} \frac{2^{N}}{\sqrt{N}}
$$

states $x$ whith $M(x)=M_{m p}$. We call these states equilibrium states.

During a cycle, the average time interval between two consecutive equilibrium states increases with $\sqrt{N}$ according to Eq. (11).

\section{MACROSCOPIC DYNAMICS}

We investigate the two following questions:

1. When the system starts from an initial state $x$ which is far away from equilibrium, for instance with $M(x)=N / 4$, how long does it take to reach an equilibrium (= most probable) state?

2. When the system starts from an equilibrium state, $M(x)=N / 2$, how long does it take to reach a nonequilibrium state with $M=N / 4$ ?

We call these two time intervals first passage time $T_{e q}$ and $T_{n e q}$, respectively. Both of these times depend on the special choice of the initial state, therefore we obtain a distribution of first passage times. Fig. 11 shows the result for $N=24$ which is obtained from simulations of all $2^{24}-1$ states. The time $T_{e q}$ to reach an equlibrium state has a sharp peak around $T_{e q} \simeq N$. In contrast, the time to leave equilibrium has a broad distribution extending from the minimal possible time $T_{n e q}=N / 4$ to $T_{n e q}=5079$. Since the system is time reversible, the 
distribution of equilibrium times should be part of the distribution of non-equilibrium times $T_{n e q}$. In fact, we observe the corresponding peak in Fig. 11. But surprisingly there are more equilibrium states which reach non equilibrium ones for the first time in short than in long times. The distribution of $T_{n e q}$ has its maximum for values of the order of $N$, while the tail can be fitted to an exponential distribution

$$
P(t) \propto e^{-t / \tau}
$$

where $\tau$ is of the order of the average non-equilibrium time $\left\langle T_{n e q}\right\rangle$, averaged over all initial equilibrium states.

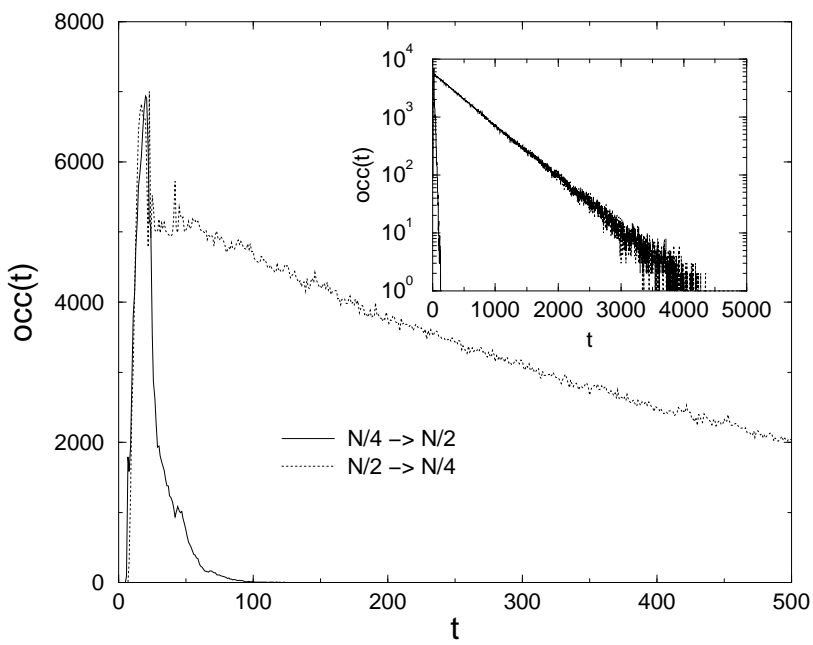

FIG. 1. Distribution of first-passage times to equilibrium $(N / 2$, solid line) and non-equilibrium $(N / 4$, dotted line $)$ in the 24-bit shift register. All states are counted; therefore the integral over the solid curve is $\left(\begin{array}{c}24 \\ 6\end{array}\right)$ while that over the dotted curve is $\left(\begin{array}{l}24 \\ 12\end{array}\right)$. The inset shows the same curves on a semi-logarithmic scale.

In Figs. 2 and 3 it is shown how the average value of these times scales with system size $N$. For small systems $N<32$ we average over all possible initial states whereas for large systems we average over several hundred randomly selected initial states. We obtain a good fit with

$$
\begin{aligned}
\left\langle T_{e q}\right\rangle & =1.30 N-12.3 \\
\left\langle T_{n e q}\right\rangle & =15.0 \exp (0.146 N) .
\end{aligned}
$$

Hence, the time to reach equilibrium is short, it scales with system size $N$, whereas the time to reach a state far from equilibrium is very long and increases exponentially with $N$. A lower bound on $\left\langle T_{n e q}\right\rangle$ can be found for any system whose distribution of macrostates is the binomial distribution: take a sequence of $\left(\begin{array}{c}N \\ N / 2\end{array}\right) /\left(\begin{array}{c}N \\ N / 4\end{array}\right)$ equilibrium states followed by one nonequilibrium state with $M=N / 4$, repeated as often as necessary. Incidentally, this lower bound differs only by a factor of $\sqrt{2 /(\pi N)}$ from the average return time of the non-equilibrium state.
Choosing an initial occupation $M_{0}=m N$, the lower bound can be approximated for large $N$ using Stirling's Formula:

$$
\left\langle T_{n e q}\right\rangle \geq \sqrt{2 m(1-m)}\left(2 m^{m}(1-m)^{1-m}\right)^{N} .
$$

For $M_{0}=N / 4$, this gives

$$
\left\langle T_{n e q}\right\rangle \geq \sqrt{3}\left(3^{3 / 4} / 2\right)^{N} \approx \sqrt{3} \exp (0.131 N)
$$

the exponent is close to the fit given in Eq. (13). Together with the upper bound $\left\langle T_{n e q}\right\rangle \leq 2^{N}-1$ one obtains exponential scaling with $N$. We find that usually $T_{n e q}$ is proportional to the return time of the non-equilibrium state, with a proportionality constant that depends on the details of the generator (for example, if a two-tap or four-tap register is chosen, see Fig. 3).

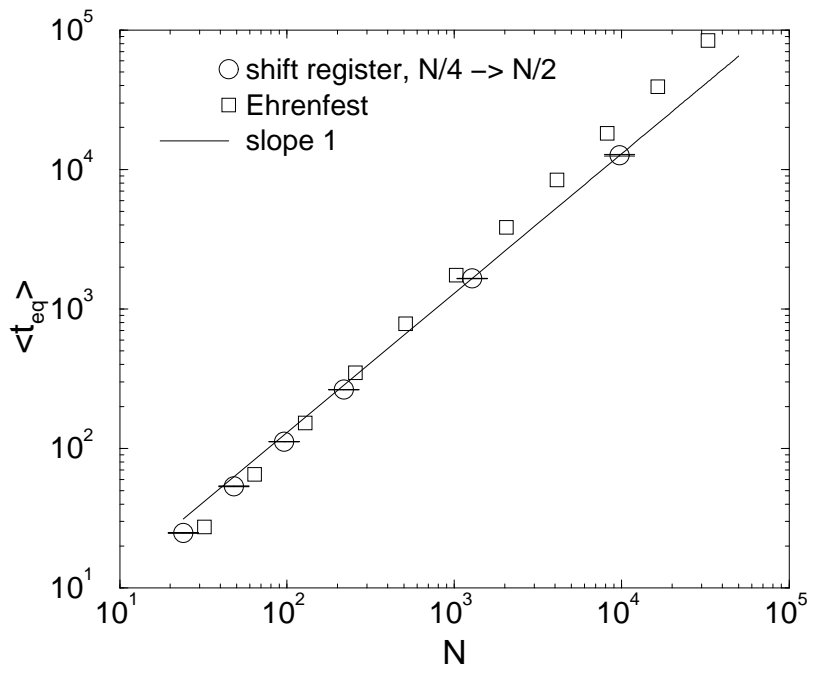

FIG. 2. Mean first-passage times from $M=N / 4$ to equilibrium for different sizes $N$ of the shift register and Ehrenfest model.

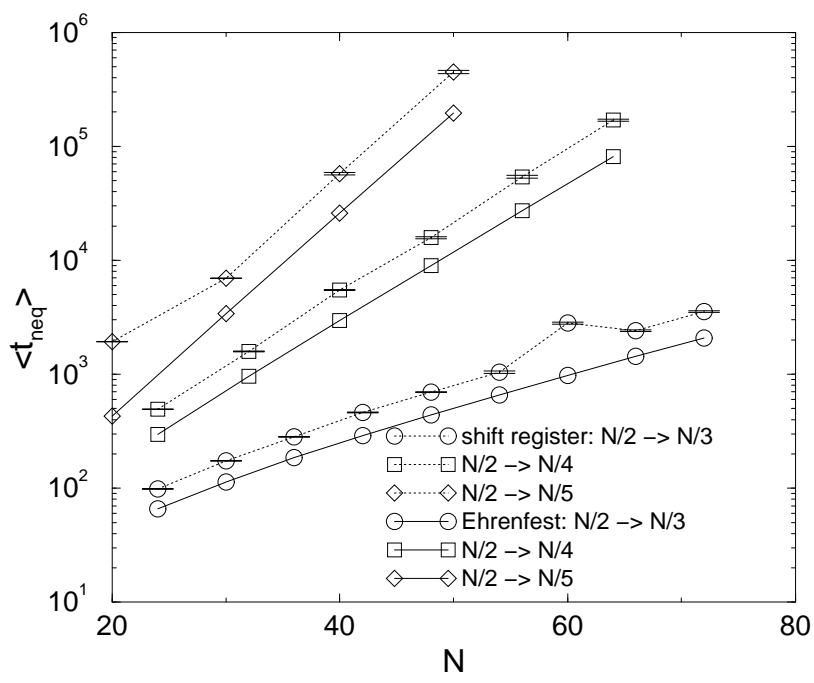


FIG. 3. Mean first-passage times from equilibrium to $M=N / 3, N / 4$ and $N / 5$, for different $N$ of the shift register and Ehrenfest model. Two-tap shift generators were used for $N=20$ and $N=60$, while the other points are from four-tap generators.

Fig. 1 shows the time dependence of the Boltzmann entropy $S_{B}(x(t))$, averaged over initial states with $M=$ $N / 4$ (non-equilibrium) and $M=N / 2$ (equilibrium), respectively. The non-equilibrium entropy decays to its equilibrium value $S_{B} \simeq S_{G}$ in $N$ time steps, since every bit $x_{i}$ is visited and "randomized" exactly every $N$ th time step. Starting from equilibrium states, the entropy stays constant. Note that after the Poincaré return time $T_{R}, S_{B}$ has to return to its initial value. But the time to reach equilibrium is of the order of the microscopic time, whereas the return time $T_{R}$ increases exponentially with system size.

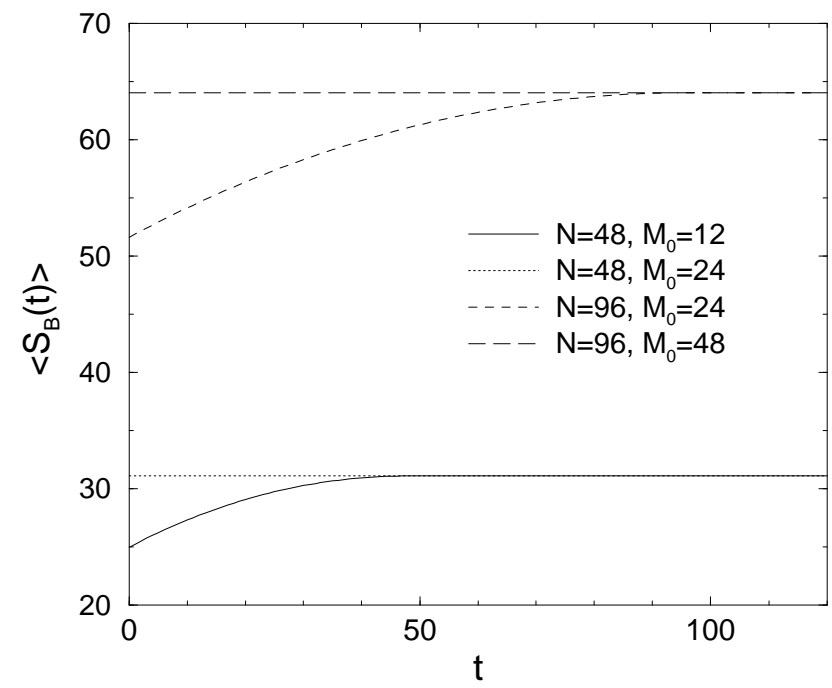

FIG. 4. Time development of the Boltzmann entropy $S_{B}$ of shift registers with $N=48$ and $N=96$. Simulations averaged over $10^{5}$ initial conditions with $M_{0}=N / 4$ and $M_{0}=N / 2$. The entropy relaxes to its maximum value in $N$ time steps.

\section{STOCHASTIC EHRENFEST MODEL}

The original Ehrenfest model describes a stochastic process: $N$ balls are distributed among two urns. At every time step, a ball is picked at random and moved to the respective other urn; $M$ is the number of balls in one of the urns. This process can be mapped to a random walk with drift [17]. $M$ is the position of the walker, who at each time step performs a step $\Delta M= \pm 1$ with probability

$$
\begin{aligned}
& P(M \rightarrow M+1)=(N-M) / M \\
& P(M \rightarrow M-1)=M / N .
\end{aligned}
$$

Hence, there is a drift towards the center $M=N / 2$. To our knowledge, analytic calculations of first passage times exist only between equilibrium states [18], whereas we are interested in the decay to or from non equilibrium states. We have calculated these times from numerical iteration of the corresponding transition matrices using absorbing states 19. The average times for different system sizes are shown in Figs. 2 and 3 in comparison with the corresponding deterministic model; the distribution of times for $N=24$ is shown in Fig. 5. Again, the decay time $T_{e q}$ to equilibrium scales with $N$ (although with more pronounced finite-size effects) while the time to reach a non equilibrium state from an equilibrium one increases exponentially with system size. Indeed the stochastic model has similar properties as the deterministic one.

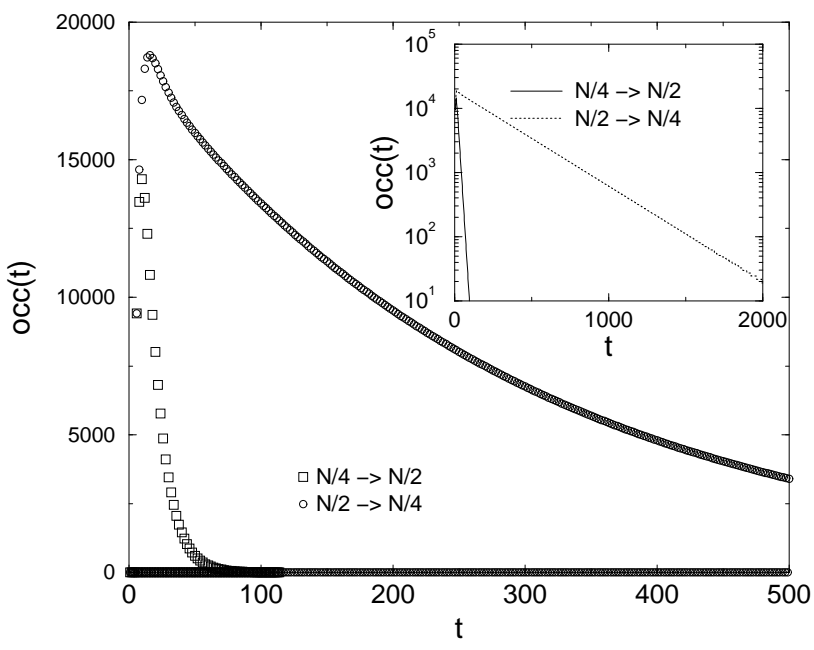

FIG. 5. Distribution of first-passage times in the stochastic Ehrenfest model, using the same normalization as in Fig. 1 .

\section{HIERARCHICAL DYNAMICS}

There are several deterministic algorithms which change only one bit per time step and run through all of the $2^{N}$ configurations of the bit sequence $x$. One of them is the Gray Code [14]: Find the largest power $2^{j}$ by which the discrete time $t$ is divisible and change bit $x_{j+1}$. The quantity $M$ changes by \pm 1 only, and the system runs through all of the $2^{N}$ possible states. However, now the access to the different variables $x_{j}$ is hierarchical; it takes $2^{j+1}$ steps before site $j$ is visited again. This hierarchical structure of microscopic dynamics leads to slow relaxation times of the macroscopic property $M$, as shown in Fig. 6. The time to reach an equilibrium state from an initial non-equilibrium one scales exponentially with system size $N$.

There is a corresponding stochastic version of the Gray code:

- Start with $j=1$;

- with probability $1 / 2$, increase $j$ by 1 ; else flip $x_{j}$, increase $t$ by one and return to the first step; 
- if $j>N$, increase $t$ and return to the first step; else repeat the second step.

The ensemble average of $M$ decays as

$$
\langle M\rangle=N / 2+\sum_{j=1}^{N}\left(M_{0} / N-1 / 2\right) \exp \left(-t / 2^{j-1}\right) \text {. }
$$

The simulations of the stochastic model agree with Eq. (17); however, in the deterministic Gray code, $\langle M\rangle$ shows structures caused by "almost returns" after times that are multiples of high powers of 2 (see Fig. 6). The first passage times of the stochastic model show a similar exponential scaling as the corresponding times of the deterministic model (see Fig. 7).

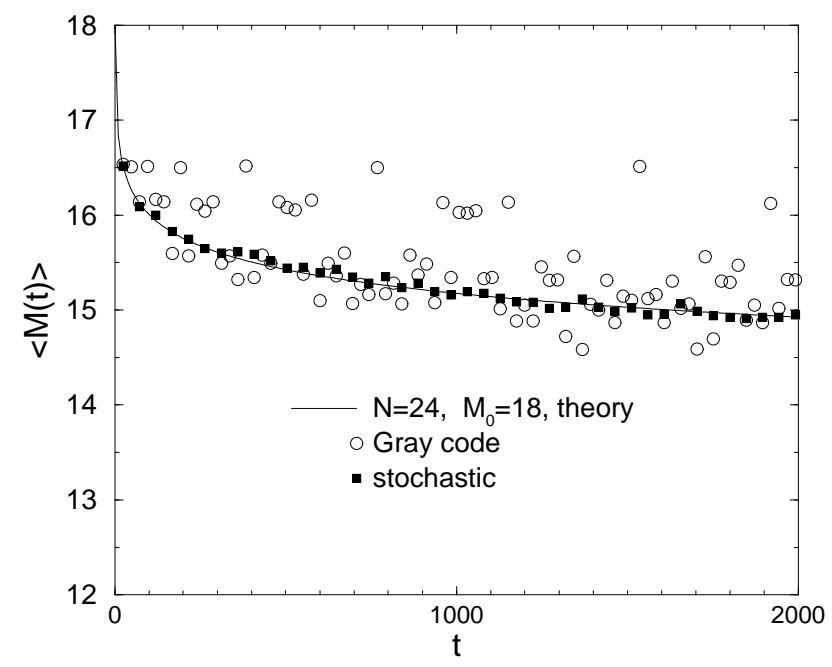

FIG. 6. Relaxation of $\langle M\rangle$ in the Gray code and corresponding stochastic model, compared to Eq. (17). Relaxation times are exponential in $N$.

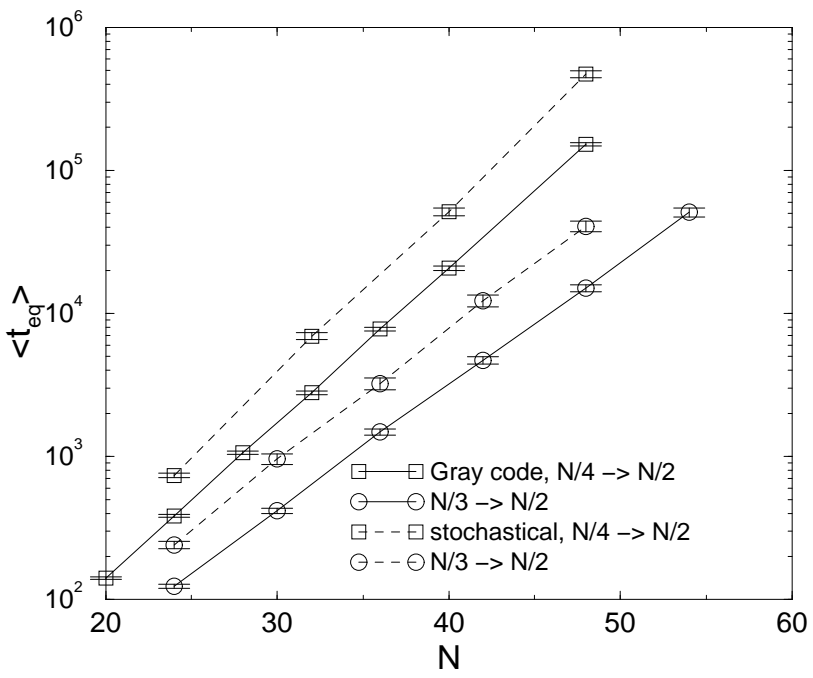

FIG. 7. In the Gray code and corresponding stochastical model, times for transition to equilibrium are exponential in $N$, with identical exponents but different prefactors.
It is possible to have a quadratic dependence on $N$ of the decay time of $\langle M(t)\rangle$ by the following stochastic algorithm:

Draw a uniform random number $r \in[0,1]$ and flip $x_{j}$ with the largest index $j<1 / r$, if $j \leq N$.

The probability to flip $x_{j}$ is of order $1 / j^{2}$. The average value of $M(t)$ decays as

$$
\begin{aligned}
\langle M\rangle \approx & N / 2+\left(M_{0} / N-1 / 2\right)\left[(N+1) \exp \left(-\frac{2 t}{(N+1)^{2}}\right)\right. \\
& \left.-\exp (-2 t)+\sqrt{2 \pi t}\left(\operatorname{erf}\left(\frac{\sqrt{2 t}}{N+1}\right)-\operatorname{erf}(\sqrt{2 t})\right)\right] .
\end{aligned}
$$

The long term behaviour is determined by the longest decay constant, which is $-2 /(N+1)^{2}$. We have also calculated the first passage time to equilibrium, and find that it scales with $N^{2}$.

\section{SUMMARY}

A simple model with time reversible deterministic dynamics is investigated. The Poincaré return time is $2^{N}-1$, where $N$ is the size of the system; each initial state returns to itself after $2^{N}-1$ time steps. We define a macroscopic quantity $M(t)$ and study its time dependence. When the system starts from an equilibrium state (state with most probable value of $M$ ) it takes on average an exponentially large time before it reaches a state far from equilibrium. On the other side, when the system starts far from equilibrium it reaches an equilibrium state after a period of the order of the microscopic time. The same is true for the corresponding Boltzmann entropy. This behaviour explains the time's arrow in macroscopic properties for a system with time reversible dynamics.

We have calculated the full distributions of these first passage times and compared them to the ones of the corresponding stochastic Ehrenfest model. Surprisingly the distribution of times the system needs to reach nonequilibrium for the first time reaches a maximum at times of order $N$ and monotonically decreases for larger times. However, the integral of $T_{n e q}$ over short times is small compared to the total number of equilibrium states. Therefore, on average the time to leave equilibrium scales exponentially with system size. If our computer needs $10^{6}$ steps per second, Eq. (15) gives the age of the universe for $N \approx 400$. Even for our simple model the return to non-equilibrium cannot be observed in reasonable time for $N$ significantly larger than 70 .

All these results hold only for a model with identical microscopic times for all of the local variables. We have also studied models with hierarchical or power law access times. In these cases the relaxation to equilibrium is slowed down. The time to reach equilibrium scales exponentially or with a power of system size, depending on the distribution of microscopic times. 


\section{ACKNOWLEDGEMENT}

All authors are grateful for financial support by the German-Israeli Foundation. This work also benefitted from a conference at the Max-Planck Institut für Physik komplexer Systeme, Dresden.

[1] J. Lebowitz, Physica A 1994, 1 (1993).

[2] J. Lebowitz, Phys. Today 46, 32 (1993).

[3] J. Lebowitz, in More Things in Heaven and Earth, edited by B. Bederson (Springer Verlag, New York, 1999), p. 581.

[4] D. Stauffer, Comp. Phys. Commun. 127, 113 (2000).

[5] V. Ambegaokar and A. Clerk, Am. J. Phys. 67, 1068 (1999).

[6] J. Lebowitz, Physica A 263, 516 (1999).
[7] I. Prigogine, Physica A 263, 528 (1999).

[8] P. and T. Ehrenfest, Phys. Zeit 27, 311 (1908).

[9] M. Kac, Probability and Related Topics in Physical Sciences (American Mathematical Soc., Providence, R.I., 1959).

[10] J. Lebowitz and H. Spohn, J. Stat. Phys. 19, 633 (1978).

[11] J. Lebowitz and H. Spohn, J. Stat. Phys. 29, 39 (1982).

[12] D. Levesque and L. Verlet, J. Stat. Phys. 73, 512 (1993).

[13] D. Knuth, Seminumerical Algorithms, Vol. 2 of The Art of Computer Programming, 2nd ed. (Addison-Wesley, Redwood City, 1981).

[14] W. Press, S. Teukolsky, W. Vetterling, and B. Flannery, Numerical Recipes in $C$, 2nd ed. (Cambridge University Press, Cambridge, 1992).

[15] M. Schroeder, Number Theory in Science and Communication (Springer-Verlag, Berlin, 1984).

[16] R. M. Ziff, Computers in Physics 12, 385 (1998).

[17] M. Kac, Amer. Math. Monthly 54, 369 (1947).

[18] A. Lipowski, J. Phys. A 30, L91 (1997).

[19] N. van Kampen, Stochastic Processes in Physics and Chemistry (Elsevier Science B.V., Amsterdem, 1992). 